\title{
The moving grid finite element method applied to biological problems
}

\author{
Anotida Madzvamuse ${ }^{1}$, Roger D.K. Thomas ${ }^{2}$, Toshio Sekimura ${ }^{3}$, Andrew J. \\ Wathen ${ }^{1}$, and Philip K. Maini ${ }^{4}$ \\ 1 Oxford University Computing Laboratory, Oxford OX1 3QD, U.K. \\ 2 Department of Geosciences, Franklin \& Marshall College, Lancaster, \\ Pennsylvania, 17604-3003, U.S.A. \\ 3 Department of Biological Chemistry, College of Bioscience and Biotechnology, \\ Chubu University, Kasugai, Aichi 487-8501, Japan. \\ 4 Centre for Mathematical Biology, Mathematical Institute, 24-29 St Giles. \\ Oxford OX1 3LB, U.K
}

\begin{abstract}
This paper seeks to present a novel numerical technique, the moving grid finite element method, to solve generalised Turing [20] reaction-diffusion type models on continuously deforming growing domains. Applications to bivalve ligaments and pigmentation colour patterns in the wing of the butterfly Papilio dardanus will be considered, by way of examples.
\end{abstract}

\section{Introduction}

It is half a century since the appearance of Turing's classical seminal paper [20] on the chemical basis of morphogenesis which gave rise to the emergence of reaction-diffusion theory in developmental biology. He considered a system of two reacting and diffusing chemicals (which he termed morphogens) and demonstrated that in the absence of diffusion, the system tends to a linearly stable uniform steady state, however, in the presence of diffusion, the system evolves, due to diffusion driven instability, to a spatially non-uniform pattern. Since then, many nonlinear reaction-diffusion models have been proposed [10] and analysed, mainly on geometrically simple fixed domains.

Nature is more complicated, however. For example, butterfly wing pigmentation patterns, animal coat markings [10] and shell pigmentation patterns [8] occur on geometrically complicated growing surfaces. Kondo and Asai [6] have shown that domain growth can play an important role in pattern formation. To compute the outcome of a pattern generator operating on a continuously deforming and growing domain requires novel applications of numerical computational methods. Of particular interest is the moving grid finite element method [1], and we employ this method to compute solutions of a general Turing system of two chemical morphogens on fixed, complicated and growing domains in one and two dimensions.

In section 2 we present the theory behind the moving grid finite element method applied to a generalised Turing reaction-diffusion model on a continuously deforming domain. Two biological applications are considered. Section 
3, analyses the essentially one-dimensional growth patterns of certain bivalve ligament. Section 4 considers the two-dimensional wing colour patterning in the butterfly Papilio darndanus. Finally, in section 5 we discuss future research.

\section{Moving grid finite element method}

We write the non-dimensional form of the two species Turing reaction-diffusion model on a continuously deforming domain $\Omega(t)$ in the form [3]:

$$
\begin{aligned}
& \frac{\partial u}{\partial t}+\nabla \cdot(\mathbf{a} u)=\gamma f(u, v)+p_{3}(u, v)+\nabla^{2} u \\
& \frac{\partial v}{\partial t}+\nabla \cdot(\mathbf{a} v)=\gamma g(u, v)+q_{3}(u, v)+d \nabla^{2} v
\end{aligned}
$$

where $u(\mathbf{x}, t)$ and $v(\mathbf{x}, t)$ are chemical concentrations at spatial position $\mathbf{x}$ and time $t$. We define $\mathbf{a}(\mathbf{x}, t)$ as the velocity field. The kinetic functions $f, p_{3}$, $g$ and $q_{3}$ describe the nonlinear reaction between the chemicals, with $p_{3}$ and $q_{3}$ bivariate cubic polynomials, with $f$ and $g$ encoding some of the familiar and often used reaction scheme:

(see website: http://web.comlab.ox.ac.uk/oucl/work/andy.wathen/software.html for more details and a freely downloadable software). The parameter values $\gamma$ and $d$ represent the reaction timescale and ratio of diffusion coefficients respectively. Typically, boundary conditions on the spatial domain are either zero flux (Neumann) or fixed (Dirichlet) or both.

The model equations (1) and (2) assume that the domain $\Omega(t)$ deforms continuously uniformly in time and this enables us to solve the system numerically by use of a moving grid finite elements [1]. We first derive an equivalent weak form over a space V. Multiplying (1) and (2) by $w \in V$ and applying Green's theorem we seek to find $u v \in V$ :

$$
\begin{aligned}
& \left(\frac{\partial u}{\partial t}, w\right)+(\nabla \cdot(\mathbf{a} u), w)=\left(\gamma f(u, v)+p_{3}(u, v), w\right)+\left(\nabla^{2} u, w\right), \\
& \left(\frac{\partial v}{\partial t}, w\right)+(\nabla \cdot(\mathbf{a} v), w)=\left(\gamma g(u, v)+q_{3}(u, v), w\right)+d\left(\nabla^{2} v, w\right),
\end{aligned}
$$

where $(u, w)=\int_{\Omega(t)} u w d \Omega(t)$ is the $L_{2}$-inner product. Let $V^{h} \subset V$ be a finite-dimensional space consisting only of simple functions depending only on finitely many parameters. The Galerkin form [12] seeks to find $u^{h}, v^{h} \in V^{h}$ such that

$$
\begin{aligned}
& \left(\frac{\partial u^{h}}{\partial t}, w^{h}\right)+\left(\nabla \cdot\left(\mathbf{a} u^{h}\right), w^{h}\right)=\left(\gamma f\left(u^{h}, v^{h}\right)+p_{3}\left(u^{h}, v^{h}\right), w^{h}\right)-\left(\nabla u^{h}, \nabla w^{h}\right) \\
& \left(\frac{\partial v^{h}}{\partial t}, w^{h}\right)+\left(\nabla \cdot\left(\mathbf{a} v^{h}\right), w^{h}\right)=\left(\gamma g\left(u^{h}, v^{h}\right)+q_{3}\left(u^{h}, v^{h}\right), w^{h}\right)-d\left(\nabla v^{h}, \nabla w^{h}\right)
\end{aligned}
$$


for all $w^{h} \in V^{h}$ where zero-flux boundary conditions have been applied. Here $u^{h}$ and $v^{h}$ are the finite element approximation to $u$ and $v$ respectively, defined as

$$
u^{h}(\mathbf{x}, t)=\sum_{i=0}^{N+1} u_{i}^{h}(t) \alpha_{i}(\mathbf{x}, \mathbf{s}(t)) \quad \text { and } \quad v^{h}(\mathbf{x}, t)=\sum_{i=0}^{N+1} v_{i}^{h}(t) \alpha_{i}(\mathbf{x}, \mathbf{s}(t))
$$

where $\mathbf{x} \in \mathbb{R}^{m}$ indicates the spatial coordinates and $\mathbf{s}(t)$ represents the moving grid in time. The time derivative of $u^{h}$ (or similarly $v^{h}$ ) is given by ([5], [2])

$$
\frac{\partial u^{h}}{\partial t}=\sum_{i=0}^{N+1}\left[\dot{u}_{i}{ }^{h}-\dot{x}_{i} u_{x}^{h}\right] \alpha_{i}(\mathbf{x}, \mathbf{s}(t))
$$

in one dimension and

$$
\frac{\partial u^{h}}{\partial t}=\sum_{i=0}^{N+1}\left[\dot{u}_{i}{ }^{h}-\left(\dot{x}_{i} u_{x}^{h}+\dot{y}_{i} u_{y}^{h}\right)\right] \alpha_{i}(\mathbf{x}, \mathbf{s}(t))
$$

in two dimensions. The effect of domain growth on the finite element formulation is to add extra terms as illustrated in (5) and (6). The spatial discretisation gives rise to a semi-discrete system of nonlinear ordinary differential equations. We use the Backward Euler finite difference scheme to discretise the ordinary differential equations in time. In one dimension the discretisation gives rise to symmetric, tridiagonal and diagonally dominant systems which can be solved using the Thomas algorithm [9]. In two dimensions we use a preconditioned Conjugate Gradient method [13].

\section{Growth patterns in bivalve ligaments}

The bivalve ligament can be defined as the uncalcified part of the bivalve shell which joins two valves dorsally (Fig 1, top panel). These ligaments typically consist of oblique, lamellar and fibrous sheets, alternating along the hinge so that their attachments on the two valves form characteristic chevron patterns (see [18], [7] for more details). In the family Arcidae, new elements are added at or near the middle of the growth zone as the ligament expands ventrally, while for the family Noetiidae, new elements are added to each end of the ligament, anteriorly and posteriorly (see Fig. 1). By solving numerically the Schnakenberg [14] reaction-diffusion model on a one-dimensional growing domain with fixed parameter values we generate a variety of patterns consistent with those observed in nature (see Fig. 2 top panel). Similar results can be obtained in two-dimensions as illustrated in Fig. 2 (bottom panel). This investigation show that the noetiid growth pattern can be derived from the chevron pattern by simply fixing the value of one of the morphogen at the centre of the domain. These results suggest that the noetiid pattern might not have evolved from a completely different parentage as previously thought. 


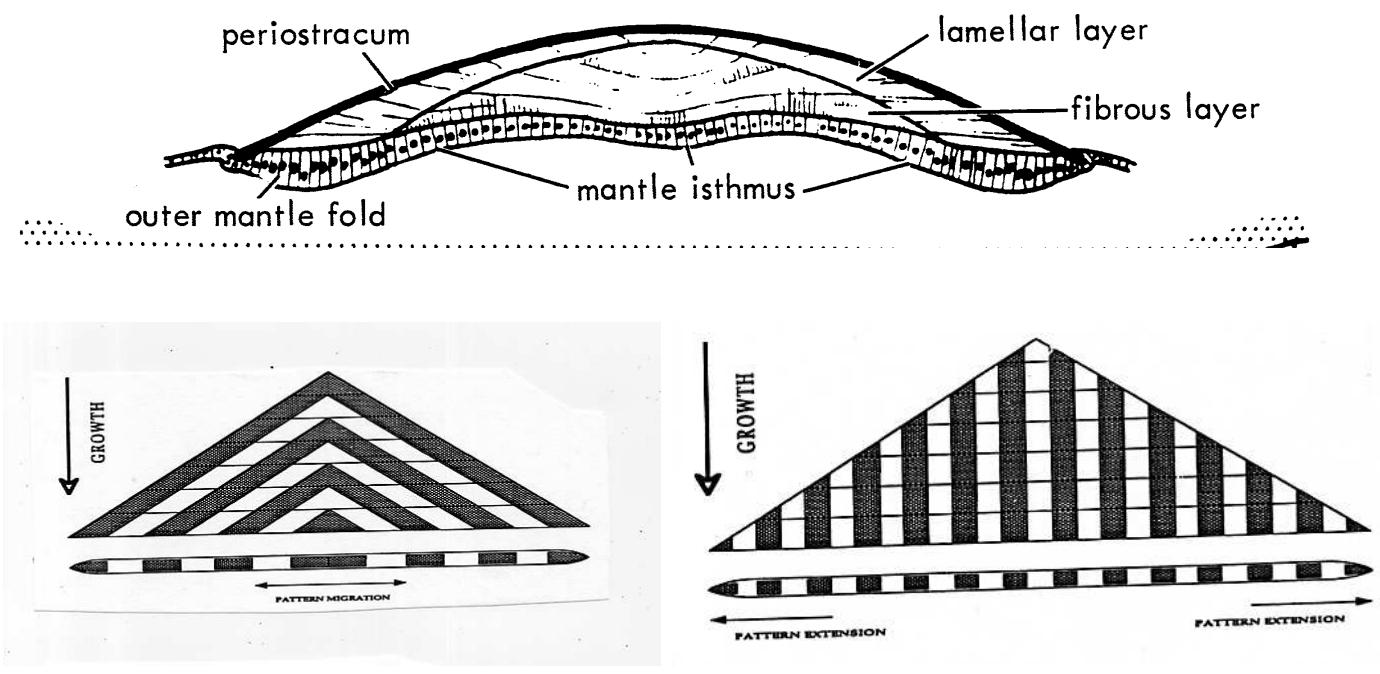

Fig. 1. Top panel: Bivalve ligament in longitudinal section. Adapted from Fig. 51, Trueman [19]. Bottom panel: Schematic showing growth patterns of the duplivincular ligament typical of glycymeris (arcoid) and the noetiid ligament showing a cross-sectional view of the ligament as it is inserted on the attachment area of each valve.

\section{Colour patterning in Papilio dardanus}

For many decades scientists have been fascinated by the spectacular colours of butterfly wing. Owing to the pioneering work of Schwanwitsch [15] and Süffert [17] on the nymphalid ground plan the seemingly complicated colour patterns on butterfly wings can now be understood as a composite of a relatively small number of pattern elements. A number of mathematical models have been put forward to account for the diversity of colour patterning (see [10], [11] for review). Nijhout [11] proposed a specific ground plan for Papilio dardanus, a species of butterfly wing known for its spectacular phenotypic polymorphism in females. Recently, Sekimura et al. [16] proposed a global wing colouration hypothesis due to stripe-like patterns of some pigment inducing morphogen. By solving the Gierer-Meinhardt [4] reaction model on a geometrically accurate (fixed) wing shape we can capture the details of the diverse colour patterns exhibited in the butterfly wing Papilio dardanus (Fig. 3) by simply modifying the gradient threshold above which pigmentation occurs. 

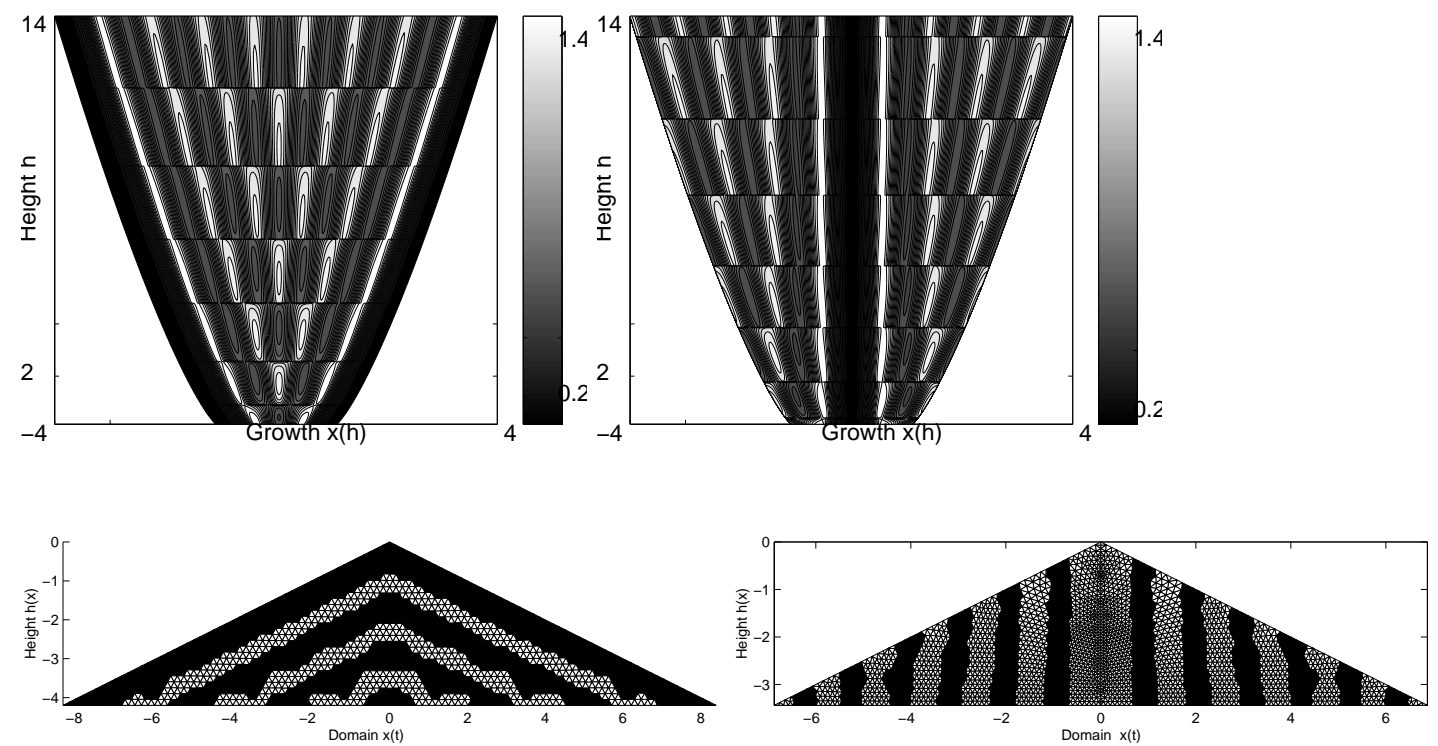

Fig. 2. Top panel: Numerically computed results for $u$ corresponding to the Schnakenberg reaction scheme with boundary conditions (a) $v=0.7$ along $x=-1$ and $x=1, u_{n}=0$ and (b) $v=0.7$ along $x=0$. (a) is consistent with arcoid patterning. (b) The resultant pattern is similar to that observed in the noetiids (compare with Fig 1 bottom panel). Bottom panel: Two-dimensional results consistent with the patterns observed in one dimension.

\section{Discussion}

The power of the moving grid finite element method applied to biological problems is that dynamically deforming complex geometries can be dealt with easily and efficiently without many changes in the numerical code. By solving the paradigm Turing model on continuously deforming domains we have shown that when a particular species exhibits morphological diversity, such diversity may arise through a simple modification of a basic ground plan. The generality of our numerical method allows us to study other biological patterns observed experimentally. We are currently carrying out computational studies of wing development in Papilio dardnus from the larval imaginal disc to the adult wing, and will use our model and numerical scheme to make predictions on the effects of experimental manipulation.

\section{Acknowledgements}

This work (AJW \& AM) was supported by the EPSRC Life Sciences Initiative grant (GR/R03914). PKM acknowledges support from a Royal Society 

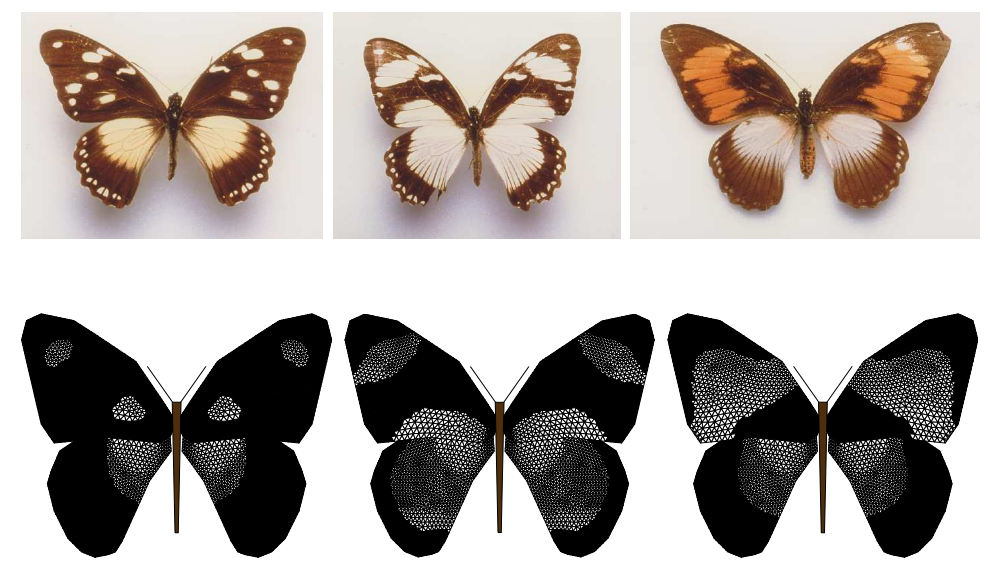

Fig. 3. Top panel: Polymorphism in females of Papilio dardanus: hippocoonides, cenea and planemoides (Courtesy of Dr. A. P. Volger and Dr. A. Cieslak of the Natural History Museum, London and Imperial College, Silwood Park). Bottom panel: Numerical results of the Gierer-Meinhardt model [4], showing patterns corresponding to hippocoonides, cenea and planemoides respectively (see [16] for full details).

Leverhulme Trust Senior Research Fellowship. This paper was written when PKM was a foreign Visiting Fellow at the Laboratory of Nonlinear Studies and Computation, University of Hokkaido, Sapporo, Japan.

\section{References}

1. Baines, M.J. Moving Finite Elements. Monographs on Numerical Analysis, Oxford: Clarendon Press, (1994)

2. Baines, M.J; Wathen, A.J. Moving finite element method for evolutionary problems. I. Theory J. Comp. Phys. 79, 245-269 (1988)

3. Crampin, E.J; Gaffney, E.A; Maini, P.K., Pattern formation through reaction and diffusion on growing domains: Scenarios for robust pattern formation, Bull. Math. Biol., 61, 1093-1120 (1999)

4. Gierer, A. Meinhardt, H. A theory of biological pattern formation. Kybernetik., 12, 30-39 (1972)

5. Jimack, P.K; Wathen, A.J. Temporal derivatives in the finite element method on continuously deforming grids. SIAM J. Numer. Anal. 28, 990-1003 (1991)

6. Kondo, S; Asai, R. A reaction-diffusion wave on the skin of the marine anglefish, Pomacanthus. Nature, 376, 765-768 (1995)

7. Madzvamuse, A; Thomas, R.D.K; Maini, P.K; Wathen, A.J. A numerical approach to the study of spatial pattern formation in the ligaments of arcoid bivalves. Bull. Math. Biol., 64, 501-530 (2002)

8. Meinhardt, H. The Algorithmic Beauty of Sea Shells, Heidelberg, New York, Springer-Verlag, (1995) 
9. Morton, K.W. and Mayers, D.F. Numerical Solution of Partial Differential Equations. Cambridge University Press, (1994)

10. Murray, J.D. Mathematical Biology. Springer-Verlag, Berlin, 2nd edition (1993)

11. Nijhout, H.F. The Development and Evolution of Butterfly Wing Patterns. Washington, DC. Smithsonian Institution Press, (1991)

12. Reddy, T.N. An Introduction to the Finite Element Method, McGraw-HIll, (1984)

13. Saad, Y. Iterative Methods for Sparse Linear Systems. PWS Publishing Co. (1996)

14. Schnakenberg, J. Simple chemical reaction systems with limit cycle behaviour. J. Theor. Biol., 81, 389-400 (1979)

15. Schwanwitsch, B.N. On the ground plan of wing-pattern in the nymphalids and certain other families of rhopalocerous Lepidoptera. Proc. Zoo. Soc. Lond. B 34, 509-528 (1924)

16. Sekimura, T; Madzvamuse, A; Wathen, A.J; Maini, P.K. A model for colour pattern formation in the butterfly wing of Papilio dardanus. Proc. Roy. Soc. Lond. B, 267, 851-859 (2000)

17. Süffert, F. Zur vergleichende analyse der schmetterlingszeichung. Biol. Zbl. 47, 385-413 (1927)

18. Thomas, R.D.K; Madzvamuse, A; Maini, P.K; Wathen, A.J. Growth patterns of noetiid ligaments: Implications of developmental models for the origin of an evolutionary novelty among arcoid bivalves. The Evol. Biol. of the Biv. Geological Soc. Lond. 177, 279-289 (2000)

19. Trueman, E.R. Ligament. In: Moore R.C. (ed.) Treatise on Invertebrate Paleontology. Geo. Soc. Amer. and Univ. of Kansas. Part N. Mollusca, 6, 58-64, (1969)

20. Turing, A. The chemical basis of morphogenesis. Phil. Trans. R. Soc. Lond. B, 237, 37-72 (1952) 